\title{
Numerical Modeling of the Influence of the Relief of a Planet on the Global Circulation of the Earth's Stratosphere and Mesosphere
}

\author{
Igor V. Mingalev, Konstantin G. Orlov, Victor S. Mingalev* \\ Polar Geophysical Institute, Russian Academy of Sciences, Murmansk Region, Russia \\ Email: *mingalev@pgia.ru
}

How to cite this paper: Mingalev, I.V., Orlov, K.G. and Mingalev, V.S. (2017) Numerical Modeling of the Influence of the Relief of a Planet on the Global Circulation of the Earth's Stratosphere and Mesosphere. Atmospheric and Climate Sciences, 7, 496-510.

https://doi.org/10.4236/acs.2017.74036

Received: August 23, 2017

Accepted: October 6, 2017

Published: October 9, 2017

Copyright $\odot 2017$ by authors and Scientific Research Publishing Inc. This work is licensed under the Creative Commons Attribution International License (CC BY 4.0).

http://creativecommons.org/licenses/by/4.0/

(c) (i) Open Access

\begin{abstract}
An investigation of the influence of the relief of a planet on the global circulation of the Earth's atmosphere is an important problem. Beyond doubt, mountains affect the global circulation of the troposphere, however, their influence on the global circulation of the stratosphere and mesosphere is not evident. In the present study, to investigate the influence of the relief of a planet on the global circulation of the Earth's stratosphere and mesosphere, the non-hydrostatic mathematical model, developed earlier in the Polar Geophysical Institute, is utilized. Calculations were made for two distinct cases. The relief of the planet was taken into account for the first case. Unlike, the Earth's surface was assumed to be smooth for the second case. Simulations were performed for the winter period in the northern hemisphere (January). Simulation results, obtained for both considered cases, are qualitatively similar at the levels of stratosphere and mesosphere, however, some noticeable distinctions exist. The horizontal domains exist, where the simulated horizontal and vertical components of the neutral wind velocity, obtained for two considered cases, differ noticeably at the levels of the stratosphere and mesosphere. Some of these horizontal domains are not connected with positions of mountains at the Earth's surface. On the contrary, some of these horizontal domains are situated above mountains.
\end{abstract}

\section{Keywords}

Numerical Simulation, Stratosphere, Mesosphere, Global Circulation

\section{Introduction}

An investigation of the planetary wind system of the Earth's atmosphere is a very 
important problem. It is well known that the human activity and health are considerably influenced by the atmospheric processes. Furthermore, cyclonic storms and hurricanes can produce tremendous damage and numerous fatalities. Unfortunately, many of the details of the formation of the planetary wind system of the Earth's atmosphere are still unresolved. To investigate features of the planetary wind system of the Earth's atmosphere not only the experimental and theoretical but also computational studies may be applied. It can be noted that several general circulation models of the Earth's lower and middle atmosphere have been developed during the last four decades (e.g., see [1]-[12]). Incidentally, the bulk of these models are hydrostatic that is the momentum equation for the vertical velocity is omitted in these models, and the vertical velocity is obtained with the help of a simple hydrostatic equation.

The study of the effect of the relief of a planet on the global circulation of the Earth's atmosphere is close to investigations of surface-atmosphere interaction (for example, see [13] [14] [15] [16]). In general, various mechanisms of the effect of Earth's surface heterogeneities on the atmospheric global circulation were discussed, in particular, dynamical and thermal effects, direct impact of the mountains on the wind, and atmospheric waves, which are generated during interaction of the upcoming flow and Earth surface.

In the present study, to investigate how the relief of a planet can affect the formation of the large-scale global circulation of the stratosphere and mesosphere, the non-hydrostatic mathematical model of the planetary wind system of the Earth's atmosphere, developed recently in the Polar Geophysical Institute (PGI), is utilized.

\section{Description of the Applied Mathematical Model}

The utilized mathematical model may be considered as a combination of two distinct mathematical models, developed earlier in the PGI. The first mathematical model, pertaining to this combination, is the non-hydrostatic model of the global neutral wind system in the Earth's atmosphere which has been described in the papers of Mingalev I. and Mingalev V. [17] and Mingalev et al. [18]. This mathematical model enables to calculate three-dimensional global distributions of the zonal, meridional, and vertical components of the neutral wind and neutral gas density over the height range from the ground up to the altitude of 120 $\mathrm{km}$. The characteristic feature of this model is that the internal energy equation for the atmospheric gas is not included in the system of governing equations. Instead, the global temperature field is supposed to be a given distribution, i.e. the input parameter of the model, and obtained from one of the existing empirical models. The first mathematical model has been applied in order to simulate the global circulation of the middle atmosphere for various geophysical conditions [17]-[24].

The second mathematical model, developed earlier in the PGI, is a limited area non-hydrostatic numerical model of the wind system of the Earth's lower 
atmosphere which has been described in the paper of Belotserkovskii et al. [25]. It can be noticed that the internal energy equation for the atmospheric gas is included in the system of governing equations of the second mathematical model. This mathematical model produces three-dimensional distributions of the atmospheric parameters in the height range from 0 to $15 \mathrm{~km}$ over a limited region of the Earth's surface. This regional mathematical model of the wind system of the lower atmosphere was earlier applied in order to investigate numerically the mechanisms responsible for the formation of large-scale vortices in the Earth's lower atmosphere [25]-[31].

It may be emphasized that both of mentioned mathematical models, developed earlier in the PGI, are non-hydrostatic numerical models of the wind system of the Earth's atmosphere. Furthermore, both of mentioned mathematical models do not take into account the relief of a planet, with the Earth's surface being smooth. On the contrary, in the mathematical model, applied in the present study, a planetary surface can contain mountains.

The mathematical model, applied in the present study, does not utilize the pressure coordinate equations of atmospheric dynamic meteorology, in particular, the hydrostatic equation. Instead, the vertical component of the air velocity is obtained by means of a numerical solution of the appropriate momentum equation, with whatever simplifications of this equation being absent. Thus, three components of the air velocity are obtained by means of a numerical solution of the generalized Navier-Stokes equation. Consequently, the applied mathematical model of the planetary wind system of the Earth's atmosphere is non-hydrostatic.

In the applied mathematical model, the atmospheric gas is considered as a mixture of air and water vapor, in which two types of precipitating water (namely, water microdrops and ice microparticles) can exist. The system of governing equations contains the equations of continuity for air and for the total water content in all phase states, momentum equations for the zonal, meridional, and vertical components of the air velocity, and energy equation. These equations are analogous to those applied in the mathematical models, described in the papers of Mingalev et al. [18] and Belotserkovskii et al. [25].

It can be noted that, in the utilized mathematical model, the internal energy equation for the atmospheric gas is written by using a relaxation approach, in which a heating/cooling rate of the atmospheric gas in various chemical-radiational processes is supposed to be straightly proportional to the difference between the real temperature of the atmospheric gas and an equilibrium temperature of the atmospheric gas. The latter equilibrium temperature may be given by utilizing the global temperature field, obtained from one of the existing empirical models, for example, from the NRLMSISE-00 empirical model [32].

In general, the applied mathematical model is based on numerical solving of non-simplified gas dynamic equations and produces three-dimensional timedependent distributions of the wind components, temperature, air density, water vapor density, concentration of micro drops of water, and concentration of ice 
particles. The model takes into account heating/cooling of the air due to absorption/emission of infrared radiation, as well as due to phase transitions of water vapor to micro drops of water and ice particles. The finite-difference method is applied for solving the system of governing equations, with numerical schemes, used in the mathematical model, having been presented in the study of Mingalev et al. [33]. The calculated parameters are determined on a uniform grid. The latitude and longitude steps are equal to $0.47^{\circ}$, and height step is equal to $200 \mathrm{~m}$. The system of gas dynamic equations is numerically solved in a simulation domain which is a layer surrounding the Earth globally. The lower boundary of this layer coincides with the Earth's surface which is approximated by an oblate spheroid, with the relief being taken into account. A planetary surface can contain mountains, it is approximated by using one of the existing digital maps of the surface relief of a planet, with the latitude and longitude steps being equal to $0.027^{\circ}$. The upper boundary of the simulation domain is the sphere lying at the altitude of $75 \mathrm{~km}$ over the equator sea level.

Detailed description of the mathematical model, applied in the present study, may be found in the paper of Chetverushkin et al. [34].

\section{Presentation and Discussion of Results}

To study how the relief of a planet can affect the formation of the large-scale global circulation of the Earth's stratosphere and mesosphere, simulation results have been obtained for two distinct cases. The relief of the planet is taken into account for the first case (Figure 1). Unlike, the Earth's surface is assumed to be smooth for the second case. It can be emphasized that, for both considered cases, the simulation results have been obtained for identical input parameters of the mathematical model. The initial moment of the calculations has corresponded to 10.30 UT for 16 January that is for winter in the northern hemisphere. At the

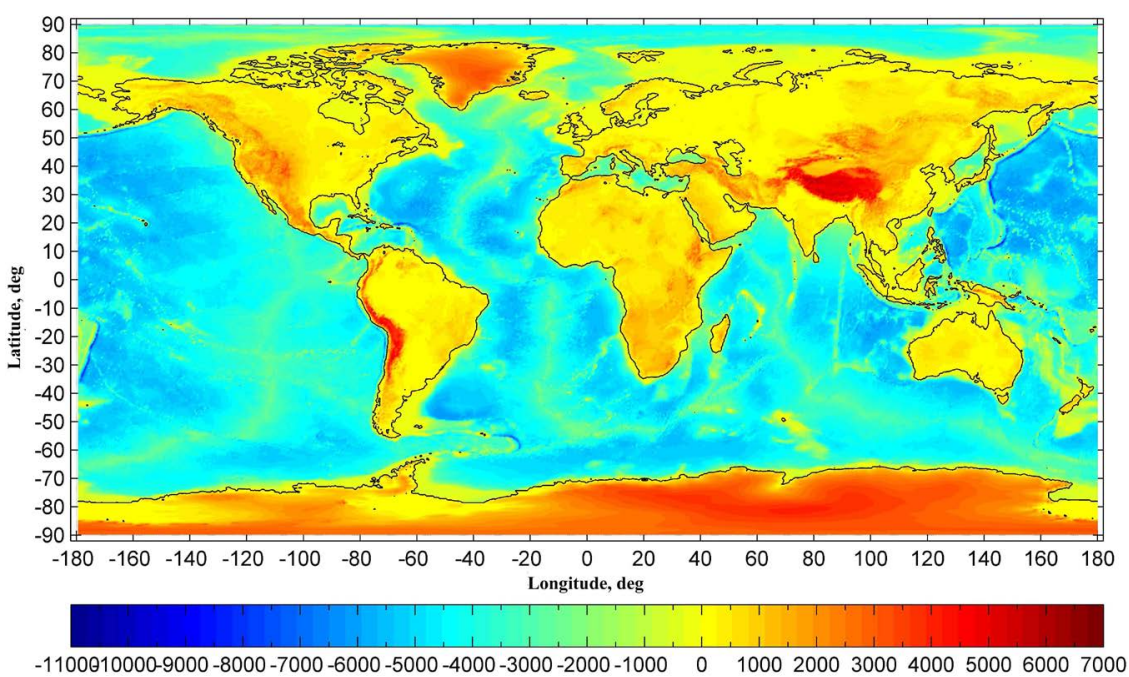

Figure 1. The utilized relief of the Earth. The colouration of the figure indicates the distance from the sea level in meters. 
initial moment, the neutral gas density at the lower boundary and air temperature in all simulation domain were taken from the NRLMSISE-00 empirical model [32], moreover, all components of the neutral wind velocity were equal to zero. The variations of the gas dynamic parameters with time were calculated during the period for more than 1000 hours.

After initial moment, three-dimensional global distributions of the gas dynamic parameters of the atmosphere, obtained for two considered cases and calculated with the help of the mathematical model, changed essentially. In the course of time, the calculated global distributions of the gas dynamic parameters of the atmosphere of the Earth acquire a tendency to fluctuate, with the period of the fluctuations being close to one day. Thus, establishing of the stable daily variations of the gas dynamic parameters came true. Consequently, daily variations of the gas dynamic parameters, conditioned by the rotation of the Earth around its axis, may be reproduced by the applied mathematical model of the global wind system.

We shall present the results of simulation, corresponding to the moment of 20.00 UT, which were obtained after the period of establishing the stable daily variations of the gas dynamic parameters. The global distributions of the gas dynamic parameters, calculated with the help of the mathematical model for two considered cases, are shown in Figures 2-7, with the coordinate systems in these figures being the same as in Figure 1. Results of simulation illustrate both common characteristic features and distinctions caused by taking into account the relief of a planet.

The global distributions of the vector of the simulated horizontal component of the neutral wind velocity at the altitude of $20 \mathrm{~km}$, obtained for both considered cases, are presented in Figure 2. The global distributions of the simulated vertical component of the neutral wind velocity at the altitude of $20 \mathrm{~km}$, obtained for both considered cases, are presented in Figure 3. The level of $20 \mathrm{~km}$ lies above a tropopause. It can be seen from Figure 2 and Figure 3 that the results of simulation, obtained for both considered cases, are very similar.

Nevertheless, some distinctions are present. Namely, the horizontal domain exists where the horizontal velocities, obtained for the case, correspondent to taking into account the relief of a planet, are more than those, obtained for the case, correspondent to smooth planetary surface. This horizontal domain is situated at latitudes close to $-60^{\circ}$ and at longitudes close to $70^{\circ}$. It turns out that, in this horizontal domain, the vertical velocities, obtained for the case, correspondent to taking into account the relief of a planet, are more than those, obtained for the case, correspondent to smooth planetary surface (Figure 3). Incidentally, archipelago Kerguelen is situated under this horizontal domain at surface of the Indian Ocean (Figure 1), with the highest mountain of the archipelago achieving the height of $1850 \mathrm{~m}$.

In the vicinity of the South Pole, the downward vertical velocities, obtained for the case, correspondent to taking into account the relief of a planet, are less than those, obtained for the case, correspondent to smooth planetary surface 

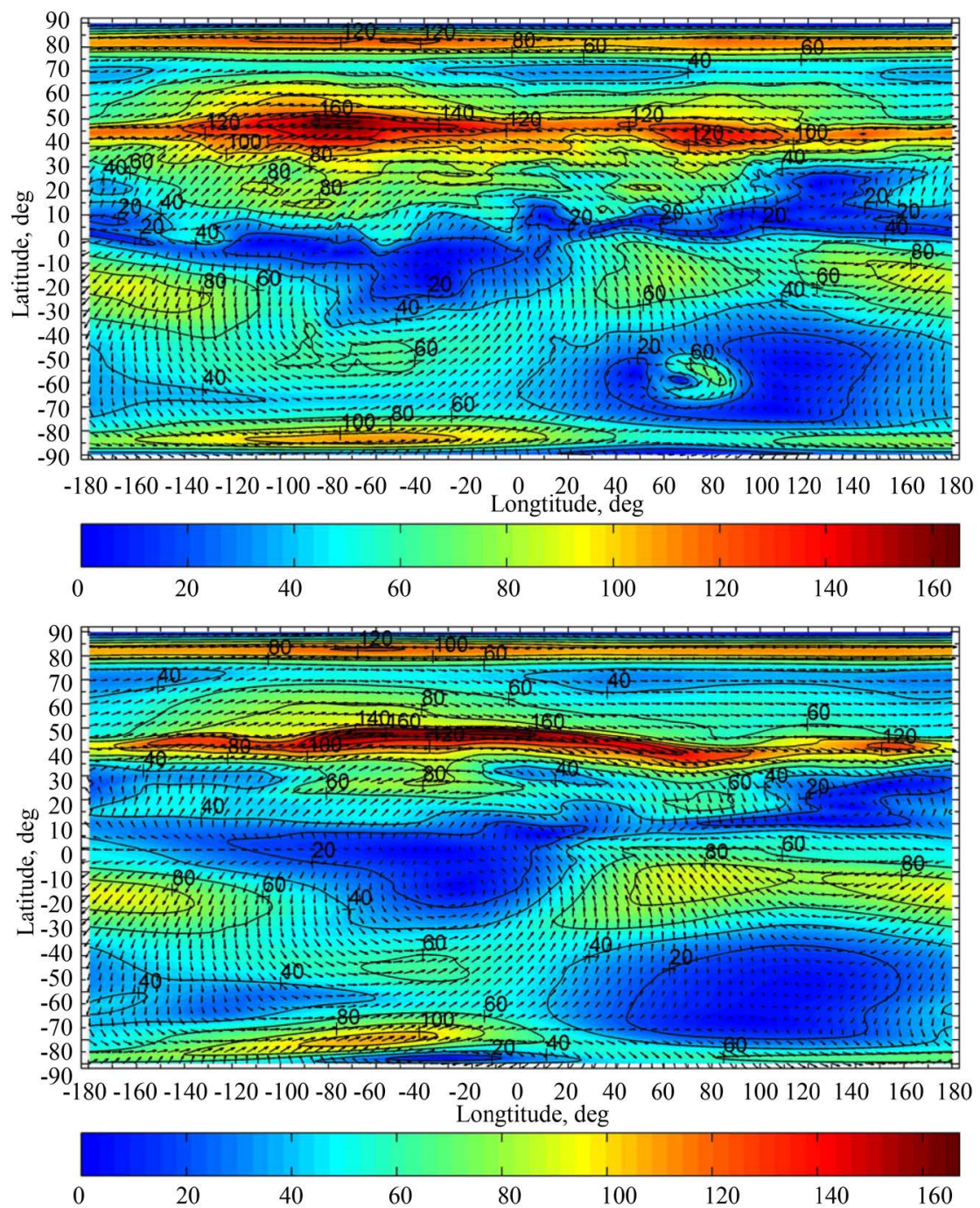

Figure 2. The global distributions of the vector of the simulated horizontal component of the neutral wind velocity at the altitude of $20 \mathrm{~km}$. The relief of the planet is taken into account (top panel) and the Earth's surface is assumed to be smooth (bottom panel). The colouration of the figures indicates the module of the velocity in $\mathrm{m} / \mathrm{s}$.

(Figure 3). This distinction may be conditioned by existence of mountains at the Antarctic (Figure 1). It is known that average height of the Antarctic is more than $2000 \mathrm{~m}$, with the highest mountain of this continent achieving the height of $5140 \mathrm{~m}$.

In Figure 4, the global distributions of the vector of the simulated horizontal component of the neutral wind velocity at the altitude of $40 \mathrm{~km}$, obtained for both considered cases, are presented. In Figure 5, the global distributions of the simulated vertical component of the neutral wind velocity at the altitude of 40 $\mathrm{km}$, obtained for both considered cases, are presented. The level of $40 \mathrm{~km}$ lies below a stratopause. From Figure 4 and Figure 5, it can be seen that the results of simulation, obtained for both considered cases, are qualitatively similar. In particular, a cyclonic vortex exists, whose center is situated at latitudes close to 

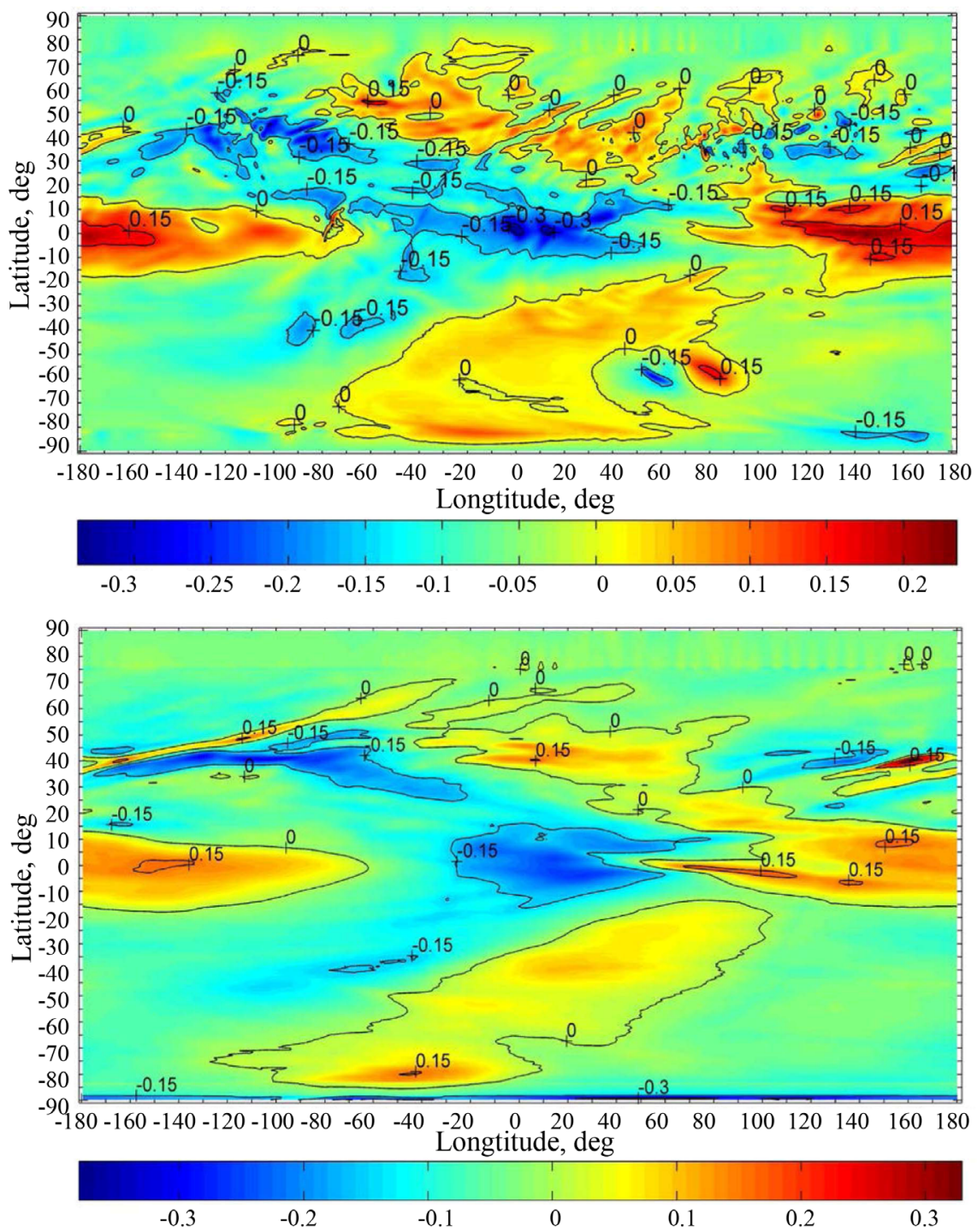

Figure 3. The global distributions of the simulated vertical component of the neutral wind velocity at the altitude of $20 \mathrm{~km}$. The relief of the planet is taken into account (top panel) and the Earth's surface is assumed to be smooth (bottom panel). The colouration of the figures indicates the velocity in $\mathrm{m} / \mathrm{s}$, with the positive direction of the vertical velocity being upward.

$-60^{\circ}$ and at longitudes close to $-110^{\circ}$, for both considered cases. Another cyclonic vortex exists, whose center is situated at latitudes close to $-60^{\circ}$ and at longitudes close to $40^{\circ}$, for both considered cases.

However, some noticeable distinctions are present. In particular, the maximal module of the horizontal wind velocity, obtained for the first case when the relief of the planet is taken into account, is more than that, obtained for the second case when the planetary surface is assumed to be smooth.

The global distributions of the calculated parameters, obtained for both considered cases at the altitude of $60 \mathrm{~km}$, are shown in Figure 6 and Figure 7. The level of $60 \mathrm{~km}$ lies in the mesosphere. It can be seen from Figure 6 and Figure 7 

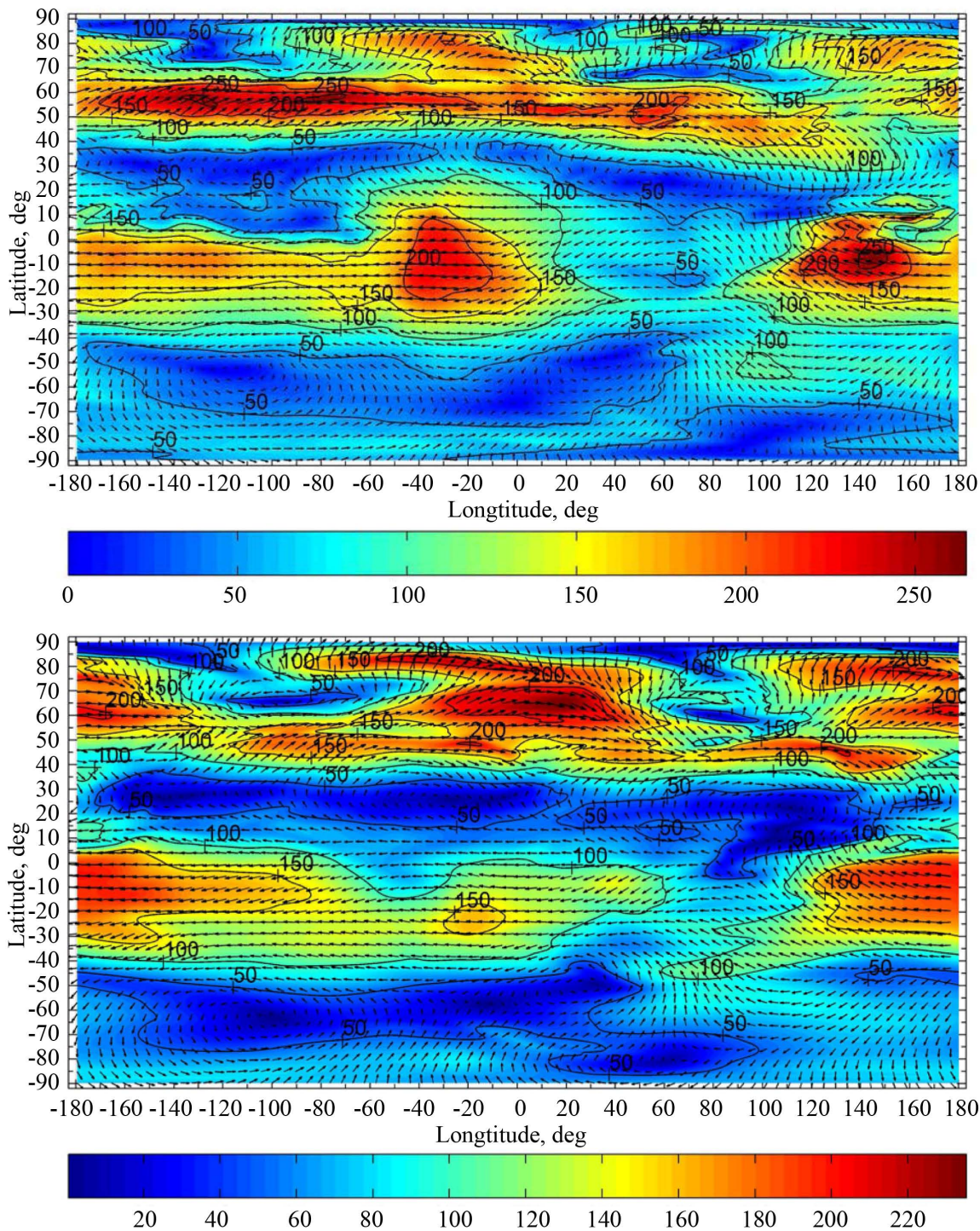

Figure 4. The global distributions of the vector of the simulated horizontal component of the neutral wind velocity at the altitude of $40 \mathrm{~km}$. The relief of the planet is taken into account (top panel) and the Earth's surface is assumed to be smooth (bottom panel). The colouration of the figures indicates the module of the velocity in $\mathrm{m} / \mathrm{s}$.

that the global distributions of the calculated parameters, obtained for both considered cases, are qualitatively similar. The horizontal domain exists where the horizontal velocities, obtained for both considered cases, have increased values. This horizontal domain is situated at latitudes close to $60^{\circ}$ and at longitudes close to $-150^{\circ}$. It turns out that, in this horizontal domain, the vertical velocities, obtained for both considered cases, are downward (Figure 7).

Distinctions of the global distributions of the calculated parameters are present in Figure 6 and Figure 7, also. In particular, the horizontal domain exists where the horizontal velocities, obtained for the first case when the relief of the planet is taken into account, have increased values. This horizontal domain is situated at latitudes close to $70^{\circ}$ and at longitudes close to $-20^{\circ}$. It turns out 

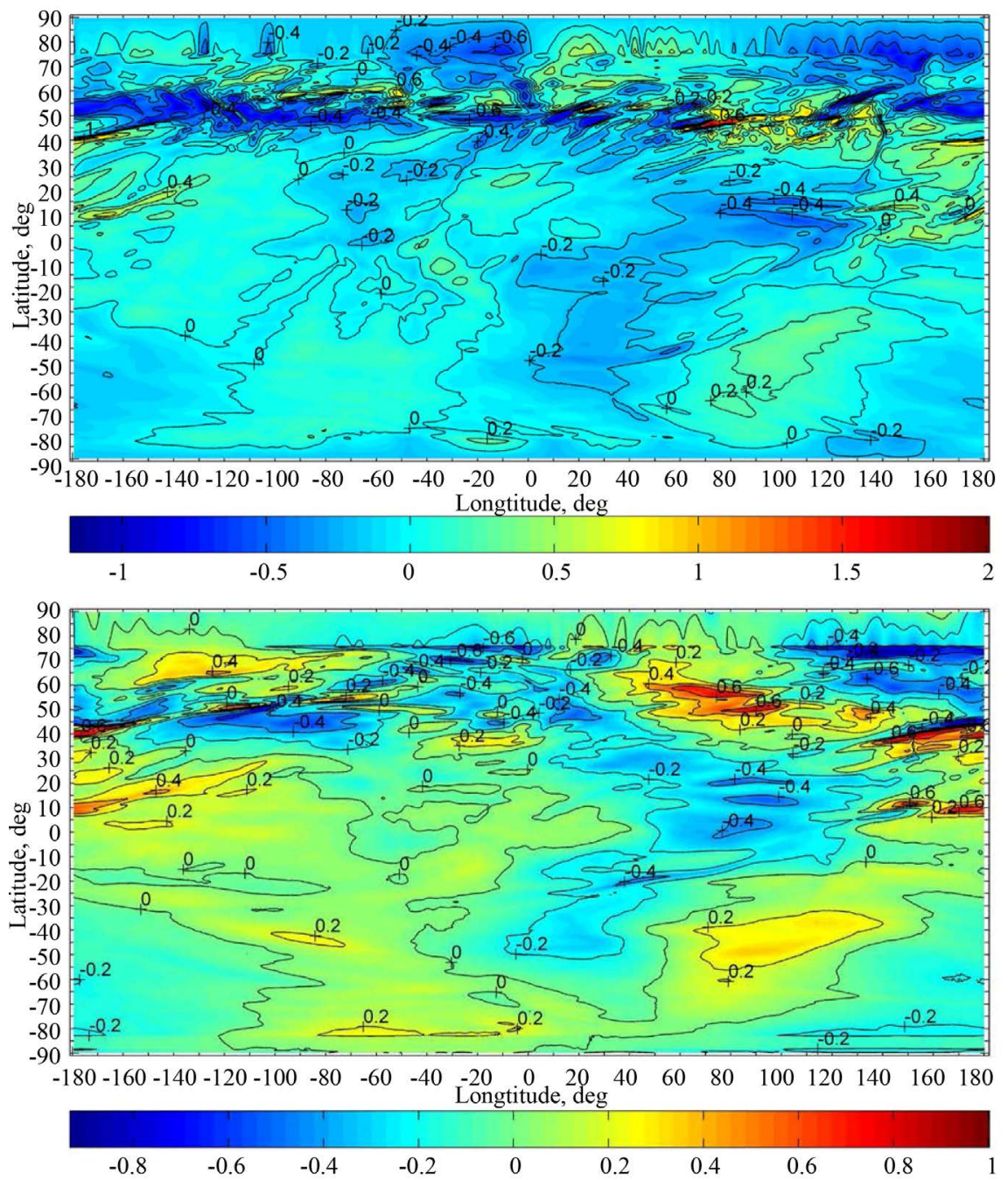

Figure 5. The global distributions of the simulated vertical component of the neutral wind velocity at the altitude of $40 \mathrm{~km}$. The relief of the planet is taken into account (top panel) and the Earth's surface is assumed to be smooth (bottom panel). The colouration of the figures indicates the velocity in $\mathrm{m} / \mathrm{s}$, with the positive direction of the vertical velocity being upward.

that, in this horizontal domain, the vertical velocities are downward. Unlike, such horizontal domain is absent for the second case when the planetary surface is assumed to be smooth. It can be noted that the biggest island Greenland is situated under this horizontal domain at the Earth's surface (Figure 1), with the highest mountain of the island achieving the height of $3694 \mathrm{~m}$. Therefore, the considered distinction between the distributions of the calculated parameters may be conditioned by existence of mountains at the Greenland.

It can be noticed that the existing hydrostatic general circulation models of the atmosphere (in particular, [1]-[11]) produce the vertical component of the wind velocity having the values of several centimeters per second at levels of the middle atmosphere and lower thermosphere. Therefore, these hydrostatic models cannot describe the global neutral wind system under disturbed conditions 

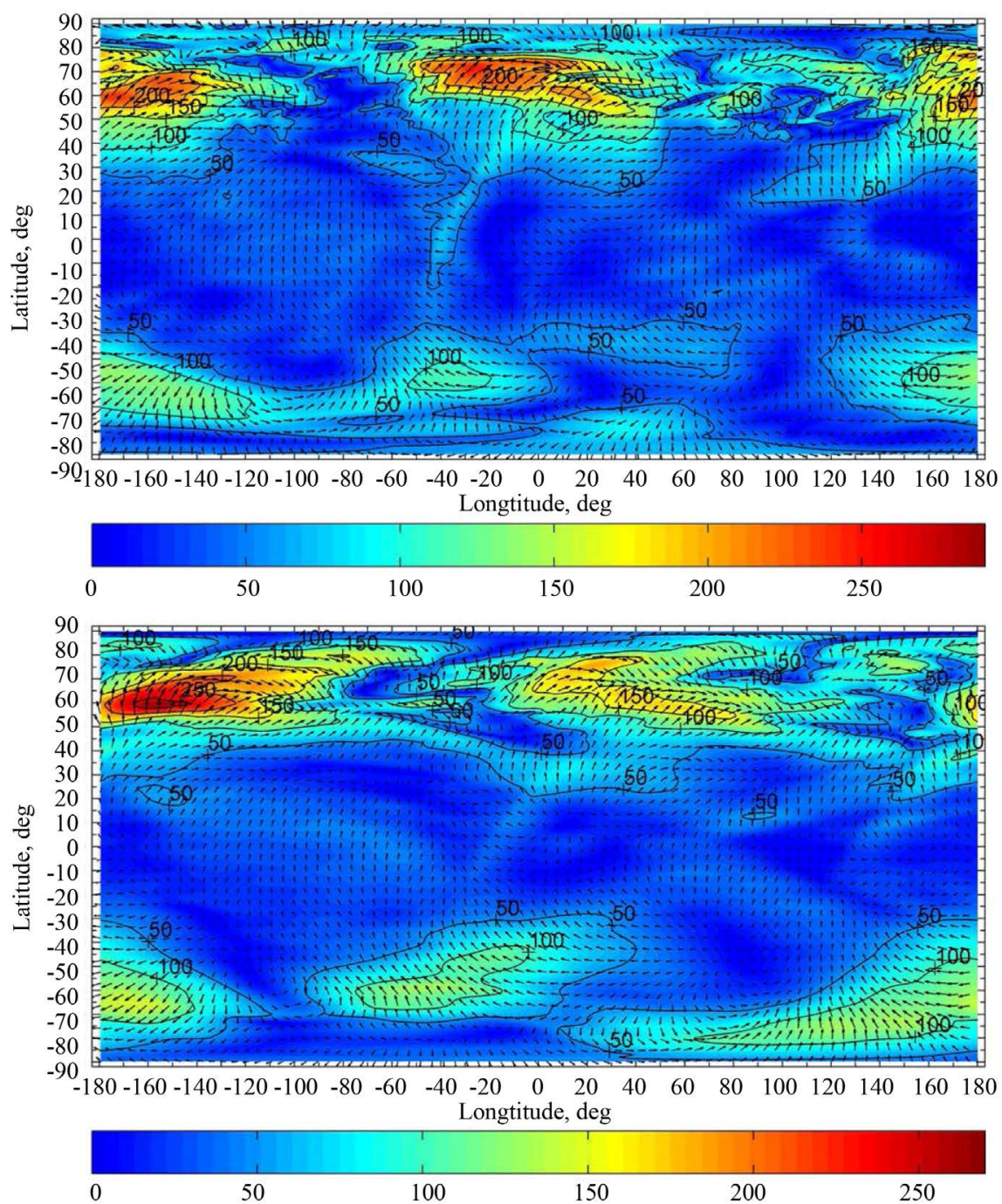

Figure 6. The global distributions of the vector of the simulated horizontal component of the neutral wind velocity at the altitude of $60 \mathrm{~km}$. The relief of the planet is taken into account (top panel) and the Earth's surface is assumed to be smooth (bottom panel). The colouration of the figures indicates the module of the velocity in $\mathrm{m} / \mathrm{s}$.

when the vertical component of the neutral wind velocity at the levels of the mesosphere and lower thermosphere can be as large as several tens of meters per second [35] [36] [37] [38] [39].

Fortunately, the mathematical model, applied in the present study, can describe the global neutral wind system under disturbed conditions, when the vertical component of the neutral wind velocity at the levels of the middle atmosphere can be rather large, due to the fact that the model is non-hydrostatic. From the simulation results, obtained in the present study, we can see that the simulated upward and downward vertical components of the neutral wind velocity can achieve values of a few $\mathrm{m} / \mathrm{s}$ at the levels of the mesosphere. Such values of the upward and downward vertical components of the neutral wind velocity are unachievable for hydrostatic general circulation models. 

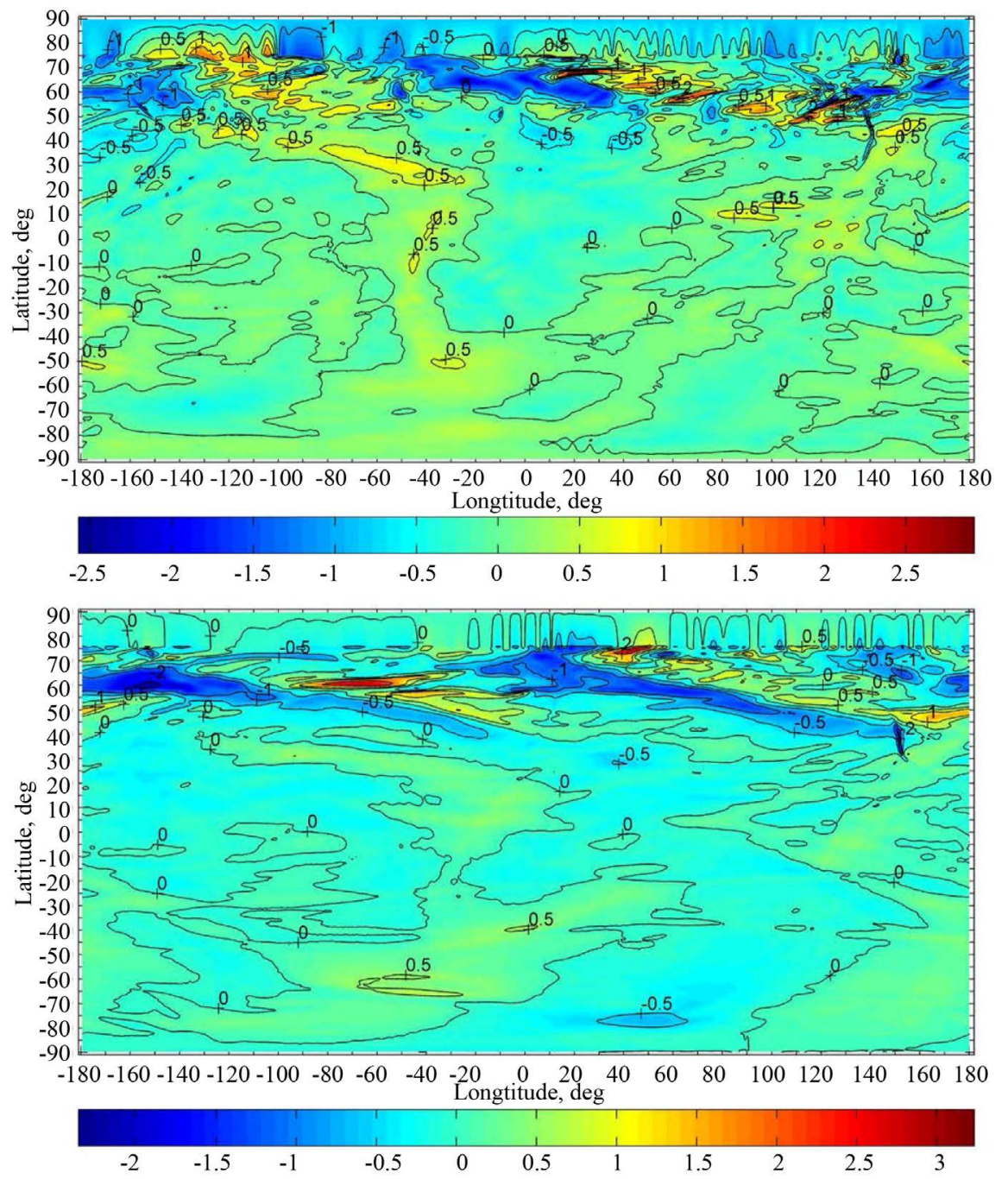

Figure 7. The global distributions of the simulated vertical component of the neutral wind velocity at the altitude of $60 \mathrm{~km}$. The relief of the planet is taken into account (top panel) and the Earth's surface is assumed to be smooth (bottom panel). The colouration of the figures indicates the velocity in $\mathrm{m} / \mathrm{s}$, with the positive direction of the vertical velocity being upward.

\section{Conclusions}

There is not a shadow of doubt that the relief of a planet influences the global circulation of the Earth's troposphere. Really, many Earth's mountains can achieve the heights of some kilometers. However, the question about influence of the relief of a planet on the global circulation of the Earth's stratosphere and mesosphere is not simple. In the present study, the mathematical model of the planetary wind system of the Earth's atmosphere, developed recently in the PGI, was applied to investigate how the relief of a planet can affect the formation of the large-scale global circulation of the stratosphere and mesosphere.

The applied mathematical model is based on the numerical solution of the system of gas dynamic equations in the layer surrounding the Earth globally and stretching from the ground up to the altitude of $75 \mathrm{~km}$. The relief of a planet is 
taken into account by the applied mathematical model. The mathematical model produces three-dimensional time-dependent distributions of the gas dynamic parameters of the atmosphere.

To investigate the influence of the relief of a planet on the global circulation of the Earth's stratosphere and mesosphere, calculations were made for two distinct cases. The relief of the planet was taken into account for the first case. Unlike, the Earth's surface was assumed to be smooth for the second case.

Simulation results indicated that the applied mathematical model of the global wind system reproduces daily variations of the gas dynamic parameters, conditioned by the rotation of the Earth around its axis. It turned out that the global distributions of the simulated horizontal and vertical components of the neutral wind velocity, obtained for both considered cases, are qualitatively similar at the levels of stratosphere and mesosphere.

However, the simulated global distributions of the gas dynamic parameters display some noticeable distinctions. At the levels of stratosphere and mesosphere, the horizontal domains exist where the simulated horizontal and vertical components of the neutral wind velocity, obtained for two considered cases, differ conspicuously. Some of these horizontal domains are not connected with positions of mountains at the Earth's surface. On the contrary, some of these horizontal domains are situated above mountains.

Thus, it was established with the help of mathematical modeling that the relief of the planet ought to influence conspicuously on the global circulation of the Earth's stratosphere and mesosphere for the winter period in the northern hemisphere.

It can be noticed that the applied mathematical model was able to simulate the noticeable effect of the relief of a planet on the global circulation of the Earth's stratosphere and mesosphere due to the fact that the model is non-hydrostatic.

\section{Acknowledgements}

This work was partly supported by Grant No. 17-01-00100 from the Russian Foundation for Basic Research. The authors would like to thank the reviewers for helpful suggestions that led to improvement in the original manuscript.

\section{References}

[1] Manabe, S. and Hahn, D.G. (1981) Simulation of Atmospheric Variability. Mon. Wea. Rev., 109, 2260-2286.

[2] Cariolle, D., Lasserre-Bigorry, A., Royer, J.-F. and Geleyn J.-F. (1990) A General Circulation Model Simulation of the Springtime Antarctic Ozone Decrease and its Impact on Mid-Latitudes. Journal of Geophysical Research, 95 D, 1883-1898. https://doi.org/10.1029/JD095iD02p01883

[3] Rasch, P.J. and Williamson, D.L. (1991) The Sensitivity of a General Circulation Model Climate to the Moisture Transport Formulation. Journal of Geophysical Research, 96 D, 13123-13137. https://doi.org/10.1029/91JD01179

[4] Graf, H.F., Kirchner, I., Sausen, R. and Schubert, S. (1992) The Impact of Up- 
per-Tropospheric Aerosol on Global Atmospheric Circulation. Annales Geophysicae, 10, 698-707.

[5] Stott, P.A. and Harwood, R.S. (1993) An Implicit Time-Stepping Scheme for Chemical Species in a Global Atmospheric Circulation Model. Annales Geophysicae, 11, 377-388.

[6] Christiansen, B., Guldberg, A., Hansen, A.W. and Riishojgaard, L.P. (1997) On the Response of a Three-Dimensional General Circulation Model to Imposed Changes in the Ozone Distribution. Journal of Geophysical Research, 102 D, 13051-13078. https://doi.org/10.1029/97JD00529

[7] Galin, V.Y. (1997) Parametrization of Radiative Processes in the DNM Atmospheric Model. Izvestiya AN, Atmospheric and Oceanic Physics, 34, 380-389 (Russian Issue).

[8] Gibelin, A.-L. and Deque, M. (2002) Anthropogenic Climate Change over the Mediterranean Region Simulated by a Global Variable Resolution Model. Climate Dynamics, 20, 327-339. https://doi.org/10.1007/s00382-002-0277-1

[9] Mendillo, M., Rishbeth, H., Roble, R.G. and Wroten, J. (2002) Modelling F2-Layer Seasonal Trends and Day-to-Day Variability Driven by Coupling with the Lower Atmosphere. Journal of Atmospheric and Solar-Terrestrial Physics, 64, 1911-1931. https://doi.org/10.1016/S1364-6826(02)00193-1

[10] Harris, M.J., Arnold, N.F. and Aylward, A.D. (2002) A Study into the Effect of the Diurnal Tide on the Structure of the Background Mesosphere and Thermosphere Using the New Coupled Middle Atmosphere and Thermosphere (CMAT) General Circulation Model. Annales Geophysicae, 20, 225-235. https://doi.org/10.5194/angeo-20-225-2002

[11] Langematz, U., Claussnitzer, A., Matthes, K. and Kunze, M. (2005) The Climate during Maunder Minimum: A Simulation with Freie Universitat Berlin Climate Middle Atmosphere Model (FUB-CMAT). Journal of Atmospheric and Solar-Terrestrial Physics, 67, 55-69.

[12] Smith, A.K., Garcia, R.R., Marsh, D.R. and Richter, J.H. (2011) WACCM Simulations of the Mean Circulation and Trace Species Transport in the Winter Mesosphere. Journal of Geophysical Research, 116, D20115.

[13] Ortland, D.A. and Alexander, M.J. (2006) Gravity Wave Influence on the Global Structure of the Diurnal Tide in the Mesosphere and Lower Thermosphere. Journal of Geophysical Research, 111, A10S10. https://doi.org/10.1029/2005JA011467

[14] Catry, B., Geleyn, J.F., Bouyssel, F., Cedilnik, J., Broo, R., Derková, M. and Mladek, R. (2008) A New Sub-Grid Scale Lift Formulation in a Mountain Drag Parameterisation Scheme. Meteorologische Zeitschrift, 17, 193-208. https://doi.org/10.1127/0941-2948/2008/0272

[15] Geller, M.A., Zhou, T. and Ruedy, R. (2011) New Gravity Wave Treatments for GISS Climate Models. Journal of Climate, 24, 3989-4002. https://doi.org/10.1175/2011JCLI4013.1

[16] Gavrilov, N.M., Koval, A.V., Pogoreltsev, A.I. and Savenkova, E.N. (2013) Numerical Simulation of the Response of General Circulation of the Middle Atmosphere to Spatial Inhomogeneities of Orographic Waves. Izvestiya AN, Atmospheric and Oceanic Physics, 49, 367-374. https://doi.org/10.1134/S0001433813040038

[17] Mingalev, I.V. and Mingalev, V.S. (2005) The Global Circulation Model of the Lower and Middle Atmosphere of the Earth with a Given Temperature Distribution. Mathematical Modeling, 17, 24-40. (In Russian) 
[18] Mingalev, I.V., Mingalev, V.S. and Mingaleva, G.I. (2007) Numerical Simulation of Global Distributions of the Horizontal and Vertical Wind in the Middle Atmosphere Using a Given Neutral Gas Temperature Field. Journal of Atmospheric and Solar-Terrestrial Physics, 69, 552-568.

[19] Mingalev, I.V., Mingalev, O.V. and Mingalev, V.S. (2008) Model Simulation of Global Circulation in the Middle Atmosphere for January Conditions. Advances in Geosciences, 15, 11-16. https://doi.org/10.5194/adgeo-15-11-2008

[20] Mingalev, I.V., Mingalev, V.S. and Mingaleva, G.I. (2012) Numerical Simulation of the Global Neutral Wind System of the Earth's Middle Atmosphere for Different Seasons. Atmosphere, 3, 213-228. https://doi.org/10.3390/atmos3010213

[21] Mingalev, I.V. and Mingalev, V.S. (2012) Numerical Modeling of the Influence of Solar Activity on the Global Circulation in the Earth's Mesosphere and Lower Thermosphere. International Journal of Geophysics, 2012, Article ID: 106035.

[22] Mingalev, I., Mingaleva, G. and Mingalev, V. (2013) A Simulation Study of the Effect of Geomagnetic Activity on the Global Circulation in the Earth's Middle Atmosphere. Atmospheric and Climate Sciences, 3, 8-19. https://doi.org/10.4236/acs.2013.33A002

[23] Mingalev, I.V., Orlov, K.G. and Mingalev, V.S. (2014) A Computational Study of the Transformation of Global Gas Flows in the Earth's Atmosphere over the Course of a Year. Open Journal of Fluid Dynamics, 4, 379-402. https://doi.org/10.4236/ojfd.2014.44029

[24] Mingalev, I.V., Orlov, K.G. and Mingalev, V.S. (2016) A Computational Study of the Effect of Geomagnetic Activity on the Planetary Circulation of the Earth's Atmosphere. Journal of Advances in Physics, 12, 4451-4459.

[25] Belotserkovskii, O.M., Mingalev, I.V., Mingalev, V.S., Mingalev, O.V. and Oparin, A.M. (2006) Mechanism of the Appearance of a Large-Scale Vortex in the Troposphere above a Nonuniformly Heated Surface. Doklady Earth Sciences, 411, 1284-1288. https://doi.org/10.1134/S1028334X06080277

[26] Belotserkovskii, O.M., Mingalev, I.V., Mingalev, V.S., Mingalev, O.V., Oparin, A.M. and Chechetkin, V.M. (2009) Formation of Large-Scale Vortices in Shear Flow of the Lower Atmosphere of the Earth in the Region of Tropical Latitudes. Cosmic Research, 47, 466-479. https://doi.org/10.1134/S0010952509060033

[27] Mingalev, I.V., Orlov, K.G. and Mingalev, V.S. (2012) A Mechanism of Formation of Polar Cyclones and Possibility of Their Prediction Using Satellite Observations. Cosmic Research, 50, 160-169. https://doi.org/10.1134/S0010952512010066

[28] Mingalev, I.V., Astafieva, N.M., Orlov, K.G., Chechetkin, V.M., Mingalev, V.S. and Mingalev, O.V. (2012) Numerical Simulation of Formation of Cyclone Vortex Flows in the Intertropical Zone of Convergence and Their Early Detection. Cosmic Research, 50, 233-248. https://doi.org/10.1134/S0010952512020062

[29] Mingalev, I.V., Astafieva, N.M., Orlov, K.G., Mingalev, V.S., Mingalev, O.V. and Chechetkin, V.M. (2013) A Simulation Study of the Formation of Large-Scale Cyclonic and Anticyclonic Vortices in the Vicinity of the Intertropical Convergence Zone. ISRN Geophysics, 2013, Article ID: 215362.

[30] Mingalev, I.V., Orlov, K.G. and Mingalev, V.S. (2014) A Modeling Study of the Initial Formation of Polar Lows in the Vicinity of the Arctic Front. Advances in Meteorology, 2014, Article ID: 970547.

[31] Mingalev, I.V., Astafieva, N.M., Orlov, K.G., Mingalev, V.S., Mingalev, O.V. and Chechetkin, V.M. (2014) Numerical Modeling of the Initial Formation of Cyclonic Vortices at Tropical Latitudes. Atmospheric and Climate Sciences, 4, 899-906. 
https://doi.org/10.4236/acs.2014.45079

[32] Picone, J.M., Hedin, A.E., Drob, D.P. and Aikin, A.C. (2002) NRLMSISE-00 Empirical Model of the Atmosphere: Statistical Comparisons and Scientific Issues. Journal of Geophysical Research, 107, Article ID: 1468.

[33] Mingalev, V.S., Mingalev, I.V., Mingalev, O.V., Oparin, A.M. and Orlov, K.G. (2010) Generalization of the Hybrid Monotone Secondorder Finite Difference Scheme for Gas Dynamics Equations to the Case of Unstructured 3D Grid. Computational Mathematics and Mathematical Physics, 50, 877-889. https://doi.org/10.1134/S0965542510050118

[34] Chetverushkin, B.N., Mingalev, I.V., Orlov, K.G., Chechetkin, V.M., Mingalev, V.S. and Mingalev, O.V. (2017) Gas Dynamic General Circulation Model of the Lower and Middle Atmosphere of the Earth. Mathematical Modeling, 29, 59-73. (In Russian)

[35] Peteherych, S., Shepherd, G.G. and Walker, J.K. (1985) Observation of Vertical E-Region Neutral Winds in Two Intense Auroral Arcs. Planet. Space Science, 33, 869-873.

[36] Widdel, H.-U. (1987) Vertical Movements in the Middle Atmosphere Derived from Foil Cloud Experiments. Journal of Atmospheric and Terrestrial Physics, 49, 723-741.

[37] Hoppe, U.P. and Hansen, T. (1988) Studies of Vertical Motions in the Upper Mesosphere Using the EISCAT UHF Radar. Annales Geophysicae, 6, 181-186.

[38] Price, G.D. and Jacka, F. (1991) The Influence of Geomagnetic Activity on the Upper Mesosphere/Lover Thermosphere in Auroral Zone. 1. Vertical Winds. Journal of Atmospheric and Terrestrial Physics, 53, 909-922.

[39] Ishii, M. (2005) Relationship between Thermospheric Vertical Wind and the Location of Ionospheric Current in the Polar Region. Advances in Polar Upper Atmosphere Research, 19, 63-70. 